\title{
Radiotherapy and Chemotherapy in T2N0 Glottic Cancer: Does Chemotherapy Improve Local Control?
}

\author{
Takashi Kosugi $^{{ }^{*}}$, Kazunori Suzuki ${ }^{1}$, Kenta Konishi ${ }^{1}$, Hotaka Nonaka ${ }^{1}$, Hiroyuki Mineta ${ }^{2}$, \\ Harumi Sakahara ${ }^{1}$
}

${ }^{1}$ Department of Radiology, Hamamatsu University School of Medicine, Hamamatsu, Japan; ${ }^{2}$ Department of Otorhinolaryngology, Hamamatsu University School of Medicine, Hamamatsu, Japan.

Email: *tkskosugi@gmail.com

Received January $20^{\text {th }}, 2013$; revised February $21^{\text {st }}, 2013$; accepted March $1^{\text {st }}, 2013$

Copyright @ 2013 Takashi Kosugi et al. This is an open access article distributed under the Creative Commons Attribution License, which permits unrestricted use, distribution, and reproduction in any medium, provided the original work is properly cited.

\begin{abstract}
Purpose: To compare concurrent chemoradiotherapy with radiotherapy in T2N0 glottic cancer. Materials and Methods: Thirty nine patients were treated with radiotherapy alone. In detail, 30 patients treated between 1985 and 1995, and 9 patients treated between 1995 and 2010 were included in the radiotherapy alone group. Between 1995 and 2010, 22 patients were treated with concurrent chemoradiotherapy. Conventional fractionation was applied in 19 patients in the radiotherapy group and in 1 patient in the chemoradiotherapy group. Hyperfractionation was applied to 20 patients in the radiotherapy group and to 21 patients in the chemoradiotherapy group. Total dose ranged from 64 Gy to 76.8 Gy in the radiotherapy group and from $66 \mathrm{~Gy}$ to $76.8 \mathrm{~Gy}$ in the chemoradiotherapy group. Cisplatin or carboplatin was administered in the chemoradiotherapy group. Results: There were no differences in the 5-year preservation rate of vocal function ( $82.8 \%$ vs $87.4 \%, \mathrm{p}=0.652)$, local control rate $(77.2 \%$ vs $92.9 \%, \mathrm{p}=0.163)$, and 5-year overall survival rate $(86.4 \%$ vs $90.2 \%, p=0.497)$ between the radiotherapy and chemoradiotherapy groups. Conclusion: In T2N0 glottic cancer, no difference was observed between radiotherapy alone and concurrent chemoradiotherapy groups.
\end{abstract}

Keywords: T2 Glottic Cancer; Radiation Therapy; Chemotherapy; Voice Preservation; Local Control

\section{Introduction}

Glottic cancer is the most common type of laryngeal cancer. It is usually diagnosed in an early stage because patients complain of hoarseness. Radiation therapy is highly recommended for the treatment of early glottic cancer because it is effective and can save vocal function [1]. Fiveyear local control rates by radiation therapy range between $80 \%-90 \%$ in T1 glottic cancer [2]. In T2N0 glottic cancer, however, the range of the local control rate by radiation therapy is $65 \%-80 \%$ [3]. Chemotherapy in addition to radiotherapy may increase the local control rate, but the benefit of adding chemotherapy has not been clearly established.

In our institute the patients with T2N0 glottic cancer had been treated with radiation therapy alone before 1995 . Concurrent chemoradiotherapy for early glottic cancer was started in 1995. This report presents the results of a retrospective study conducted to determine whether the addition of chemotherapy improved local control, sur-

\footnotetext{
"Corresponding author.
}

vival, and preserved vocal function in T2N0 glottic cancer patients who underwent radiotherapy.

\section{Materials and Methods}

\subsection{Patients}

Sixty-one patients with T2N0 glottic cancer were treated with radiation therapy between 1985 and 2010. The histopathology was squamous cell carcinoma in all cases. All of the 30 patients who were treated between 1985 and 1995 underwent radiotherapy alone. Nine patients of age 86 or more, or with less than $60 \mathrm{ml} / \mathrm{min}$ for the creatinine clearance who were treated between 1995 and 2010, were also included in the group of radiotherapy alone. Twentytwo patients of age 85 or less with more than $60 \mathrm{ml} / \mathrm{min}$ of creatinine clearance were treated with chemoradiotherapy between 1995 and 2010. Patients' characteristics are shown in Table 1.

The UICC staging system of 1985 and subsequently updated versions were adopted for treating the patients. Although minimal differences exist in different versions, 
stages of the patients analyzed in the present study did not change by using different versions of the UICC staging systems. The latest version used in this study was the UICC 6th edition [4]. Tumor extended to the supraglottis in 37 patients, to the subglottis in 18 patients, and to both supraglottis and subglottis in 5 patients. One patient had an impaired mobility of the vocal cord. The median follow-up duration was 84.2 months ranging from 1.8 to 259.8 months. Performance status was $0-1$ in all patients [5].

\subsection{Radiation Therapy}

${ }^{60}$ Co gamma-ray was used in 34 patients before 1998 and 4 MV X-ray in 27 patients after 1999. Radiation was performed with parallel-opposed fields without elective irradiation for neck lymph nodes. The initial field size ranged from $36-112 \mathrm{~cm}^{2}$ (median $48 \mathrm{~cm}^{2}$ ). The field was reduced during the treatment period to $25-42 \mathrm{~cm}^{2}$ (median $30 \mathrm{~cm}^{2}$ ). The dose was prescribed at the isocenter in the middle of the planning target volume. Wedge filters were used to achieve homogenous dose distribution. Conventional fractionation of daily $2 \mathrm{~Gy}$ in one fraction

Table 1. Patients' characteristics.

\begin{tabular}{|c|c|c|}
\hline Characteristics & Radiotherapy alone & Chemoradiotherapy \\
\hline Number of patients & 39 & 22 \\
\hline \multicolumn{3}{|l|}{ Age } \\
\hline Median & $67 \mathrm{y}$ & $66 \mathrm{y}$ \\
\hline Range & $35-91 y$ & $47-85 y$ \\
\hline \multicolumn{3}{|l|}{ Performance status } \\
\hline $0-1$ & 39 patients & 22 patients \\
\hline \multicolumn{3}{|l|}{ Fraction } \\
\hline Once a day & 19 patients & 1 patient \\
\hline Twice a day & 20 patients & 21 patients \\
\hline \multicolumn{3}{|l|}{ Dose } \\
\hline Median & $70.2 \mathrm{~Gy}$ & 76.8 Gy \\
\hline Range & 64 - 76.8 Gy & 66 - $76.8 \mathrm{~Gy}$ \\
\hline \multicolumn{3}{|l|}{ Beam } \\
\hline${ }^{60}$ Co gamma-ray & 32 patients & 2 patients \\
\hline 4 MV X-ray & 7 patients & 20 patients \\
\hline \multicolumn{3}{|l|}{ Overall treatment time } \\
\hline Median & 47 days & 46.5 days \\
\hline Range & 40 - 59 days & 43 - 55 days \\
\hline \multicolumn{3}{|l|}{ Follow-up period } \\
\hline Median & 86.9 months & 51.7 months \\
\hline Range & 13.4 - 259.8 months & 1.8 - 158.3 months \\
\hline
\end{tabular}

was applied for 20 patients. Hyperfractionation of $1.2 \mathrm{~Gy}$ per fraction, twice a day, was applied for 41 patients. Total dose ranged from 64 Gy to 76.8 Gy in the radiotherapy group and from 66 Gy to 76.8 Gy in the chemoradiotherapy group. Overall treatment period was between 40 days and 59 days. All radiation therapy was performed in 5 days per week.

\subsection{Chemotherapy}

Chemotherapy was introduced in 1995 for selected patients of age 85 or less and creatinine clearance of 60 $\mathrm{ml} / \mathrm{min}$ or more. Twenty two patients were treated with concurrent chemoradiotherapy. One patient of age 86 or more and 8 patients with creatinine clearance of $60 \mathrm{ml} /$ min or less were excluded from the chemoradiotherapy regimen. Of the 22 patients, 9 patients were administered $5 \mathrm{mg}$ of cisplatin daily on the same day as radiation. Thirteen patients were administered carboplatin once a week, for a maximum of 7 weeks during the radiotherapy period. The dose of carboplatin was determined by the method of Calvert, et al. [6]. The target area under the free carboplatin plasma concentration versus time curve was set at 1.25 .

The historical changes in protocols of radiotherapy and chemotherapy are summarized in Figure 1.

\subsection{Statistical Analysis}

Toxicity was evaluated using Common Terminology Criteria for Adverse Events v4.0 (CTCAE 4.0) [7]. Recurrence was confirmed by histopathological examination of biopsy specimens.

The statistical analyses were performed using $\mathrm{R}$ software, version 2.14.0 [8]. The endpoints were local control, survival, and preservation of vocal function. The voice preservation group included the patient who could speak after partial laryngectomy. The first day of treatment was regarded as the starting point. Curves were calculated using the Kaplan-Meier method, and log-rank tests were used to determine statistical significance [9]. In addition to comparing concurrent chemoradiotherapy and radiotherapy alone, the differences between conventional fractionation and hyperfractionation, and between ${ }^{60} \mathrm{Co}$ gamma-ray therapy and $4 \mathrm{MV} \mathrm{X}$-ray therapy about

\begin{tabular}{|c|c|c|c|c|}
\hline \multicolumn{2}{|c|}{1985} & 95 & \multicolumn{2}{|c|}{2003} \\
\hline Chemotherapy & RT alone & $\mathrm{CDL}$ & & CBDCA weekly \\
\hline \multirow{3}{*}{ Beam } & \multicolumn{4}{|c|}{1998} \\
\hline & \multicolumn{2}{|c|}{${ }^{60}$ Co gamma-ray } & \multicolumn{2}{|c|}{ 4MV X-ray } \\
\hline & 1990 & 1997 & & \\
\hline Fractionation & $66 \mathrm{~Gy} / 33 \mathrm{fr}: 72 \mathrm{G}$ & $60 \mathrm{fr}$ & \multicolumn{2}{|c|}{$76.8 \mathrm{~Gy} / 64 \mathrm{fr}$} \\
\hline
\end{tabular}

Figure 1. Historical changes of radiotherapy and chemotherapy protocol. Abbreviations: RT: radiotherapy; CDDP: ciplatin; CBDCA: carboplatin; fr: fraction. 
preservation of vocal function were analyzed.

\section{Results}

For all 61 patients, local recurrence was seen in 10 patients and the 5-year local control rate was $82.0 \%$. Four patients died of glottic cancer progression and 1 patient died of aspiration pneumonia that may be related to chemoradiotherapy. Seven patients died unrelated to glottic cancer, and 1 patient died of unknown cause. Thus, 13 patients had died by the end of the analysis. The 5-year overall survival rate was $87.2 \%$.

Eight patients lost vocal function and the 5-year rate of preserving vocal function was $84.2 \%$. Eight patients including 6 of 10 patients who developed local recurrence, 1 patient with recurrent tumor in the thyroid and 1 patient with aspiration pneumonia underwent total laryngectomy. In 1 of 4 patients who developed local recurrence but did not undergo total laryngectomy, partial laryngectomy was performed because the recurrent tumor was carcinoma in situ. In the other 3 patients, neither total nor partial laryngectomy was performed and their vocal functions were preserved. One died of glottic cancer progression. This patient was 90 years old at the time of recurrence. One had an advanced lung cancer and died of the lung cancer. The last one was lost in the follow-up survey 24 months after the local recurrence. A summary of these patients with local recurrence and lost vocal function is shown in Figure 2.

Loss of vocal function was observed in 6 of 39 patients with radiotherapy alone and in 2 of 22 patients with concurrent chemoradiotherapy. The 5-year voice preserving rates of radiotherapy alone and concurrent

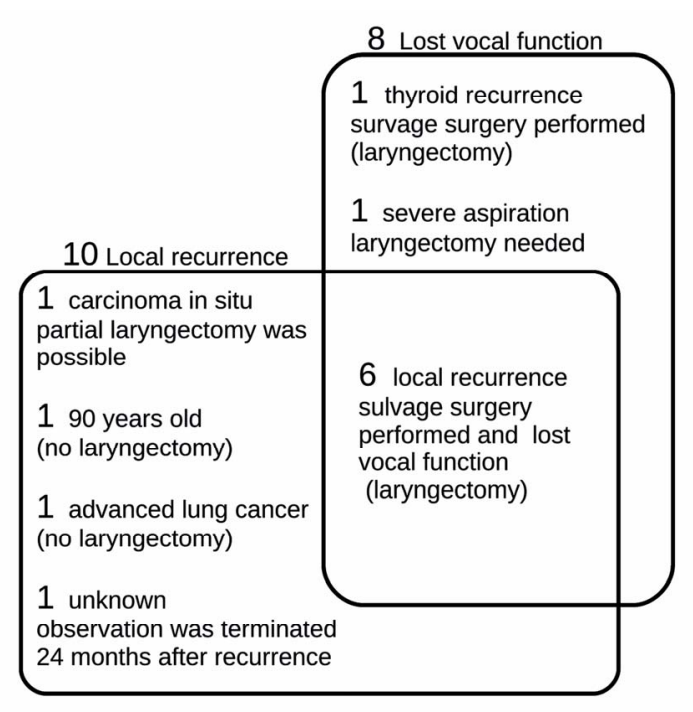

Figure 2. Cases of local recurrence and loss of vocal function. Two large squares mean local recurrence and loss of vocal function. Number of patients and reasons are shown in the squares. chemoradiotherapy were $82.8 \%$ and $87.4 \%$, respectively, and the difference lacked significance $(\mathrm{p}=0.652$, Figure 3(a)). The 5-year local control rates were $77.3 \%$ and $92.9 \%$, and the 5-year rates of overall survival were $86.4 \%$ and $90.2 \%$, respectively for groups of radiotherapy alone and concurrent chemoradiotherapy, respecttively. There were no differences between the two groups in local control and survival (Figures 3(b) and (c)).

No differences were also observed in the preservation rate of vocal function between conventional fractionation and hyperfractionation, and between ${ }^{60} \mathrm{Co}$ gamma-ray therapy and $4 \mathrm{MV}$ X-ray therapy (Figures $\mathbf{4 ( a )}$ and (b)).

Two patients developed severe complications. One patient of age 85 years died of severe pneumonia just after the chemoradiotherapy. This patient was therapy related death. The other patient in the radiation alone group was suffered from aspiration pneumonia repeatedly 3 months after the end of therapy. This patient underwent total laryngectomy but no cancer cells were seen in the resected specimen.

\section{Discussion}

No difference in local control and voice preservation was seen between radiotherapy alone and concurrent chemoradiotherapy in T2N0 glottic carcinoma. Because this study was performed on a small number of patients, the question of the efficacy of concurrent chemoradiotherapy remains unanswered, and should be pursued in a larger number of patients. Akimoto et al. reported that concurrent chemoradiotherapy yielded significant improvement in disease free survival rate over radiotherapy alone in T2N0 laryngeal cancer [10]. However, in addition to glottic cancer, this report included patients with other laryngeal cancers. Our analysis was focused on glottic cancer. No difference was seen in our study for local control, survival, and preservation of vocal function in T2N0 glottic cancer. Several studies reported good results in concurrent chemoradiotherapy for glottic cancer, but they were single arm trials and were not compared with radiotherapy alone [11-13].

In the present study the patients were placed into two groups according to the historical period. All of the 30 patients before 1995 were in the group of radiation therapy alone. Twenty two of 31 patients after 1995 underwent chemoradiotherapy and 9 patients underwent radiotherapy alone. Although the present study was not a randomized control study, the bias in categorization of patients was small. Furthermore, the radiation field in glottic cancer has remained unchanged between 1985 and 2010.

In the category of $\mathrm{T} 2$ glottic cancer, there is a remarkable variation in the volume of tumor for each patient. Several studies indicate the probability that local control is closely related to the volume of lesion $[14,15]$. The 

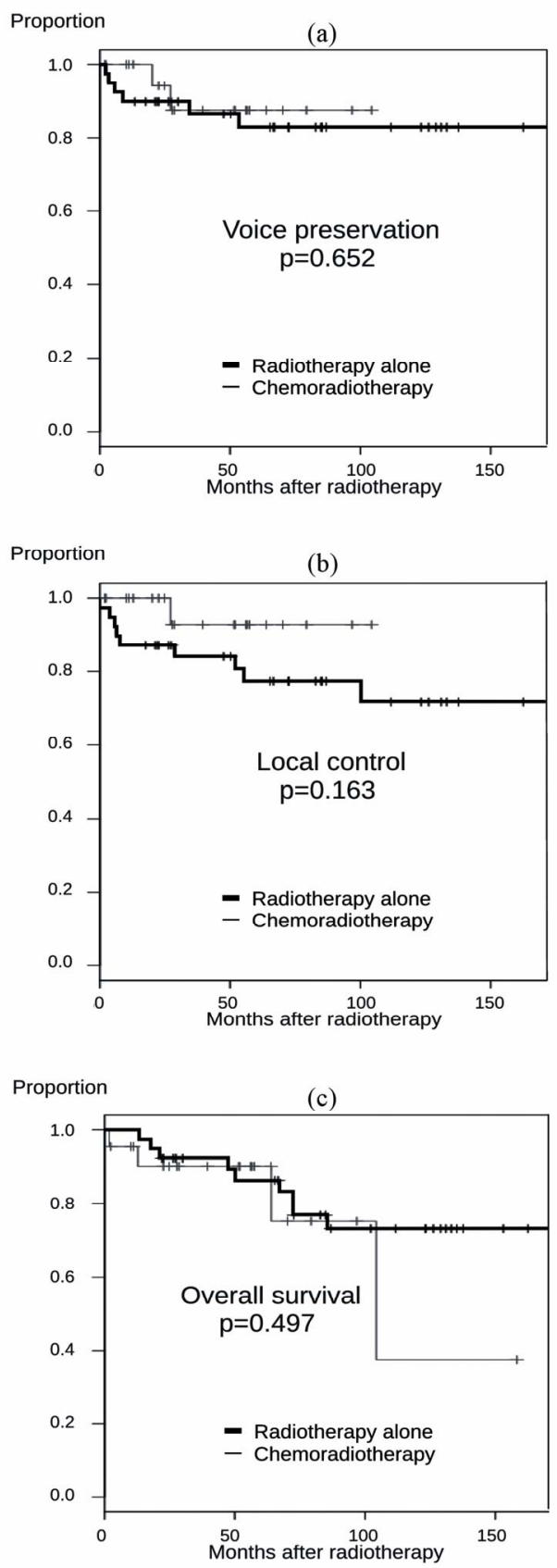

Figure 3. (a) The 5-year voice preservation rates of radiotherapy alone and concurrent chemoradiotherapy were $82.8 \%$ and $87.4 \%$, respectively, and the difference was not significant ( $p=0.652)$; (b) The 5-year local control rates of radiotherapy alone and concurrent chemoradiotherapy were $77.3 \%$ and $92.9 \%$, respectively, and the difference was not significant $(p=0.163)$; (c) The 5-year overall survival rates of radiotherapy alone and concurrent chemoradiotherapy were $86.4 \%$ and $90.2 \%$, respectively, and the difference was not significant $(p=0.497)$.

local control rate depends on the tumor volume in glottic cancer, and in T3 glottic cancer concurrent chemoradiotherapy is recommended. McCoul et al. reported that local control was significantly poor in a group with impaired vocal cord mobility [16]. Thus, further stratification of heterogeneous T2 patients may be needed to clarify the merit of adding chemotherapy.

There were several limitations with this retrospective study. First, the radiotherapy protocol and chemotherapy regimen was not standardized. ${ }^{60} \mathrm{Co}$ gamma-ray and 4 MV X-ray, conventional fractionation and hyperfractionation, and cisplatin and carboplatin were intermingled. It has been suggested that hyperfractionation improves local control in laryngeal cancer [17]. But in this study, no difference was observed in the voice preservation rate between the hyperfractionation and conventional fractionation groups. Patients treated with hyperfractionated radiotherapy overlapped those who underwent chemoradiotherapy. This made it difficult to analyze the effect of hyperfractionation independently. However, it is unlikely that the effects of hyperfractionated radiotherapy and chemotherapy are canceled by each
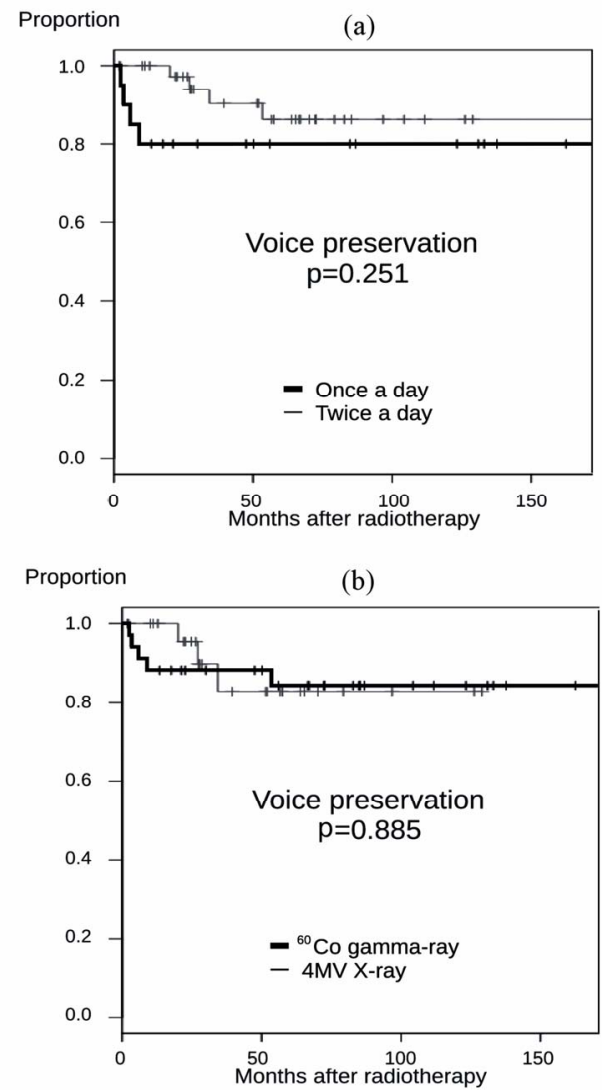

Figure 4. (a)The 5-year voice preservation rates of once a day radiotherapy and twice a day hyperfractionated radiotherapy were $80.0 \%$ and $86.3 \%$, respectively, and the difference was not significant $(p=0.251)$; (b) The 5 -year voice preservation rates of ${ }^{60} \mathrm{Co}$ gamma-ray radiotherapy and linear $4 \mathrm{MV}$-X ray radiotherapy were $84.4 \%$ and $82.9 \%$, respectively, and the difference was not significant $(p=$ 0.885). 
other. Second, acute toxicities were not fully examined. Grade 4 or more severe toxicities were included but minor toxicities were not analyzed.

The effect of salvage operation needs to be considered. If a local recurrence occurred, an appropriate surgical procedure can preserve vocal function. Local recurrence did not always lead to loss of vocal function. In our study, 10 local recurrences were observed, but only 6 patients lost vocal function.

Our chemotherapy regimen has room for further consideration. Many regimens using platinum-based-drugs and 5-fluorouracil derivatives have been proposed in laryngeal cancer. We adopted cisplatin and carboplatin but the dose was low. More powerful regimens might make concurrent chemoradiotherapy more effective than radio-therapy alone $[13,18]$.

In conclusion, in T2N0 glottic cancer, no difference was observed between radiotherapy alone and chemoradiotherapy in local control, survival, and preservation of vocal function, although the chemoradiotherapy yielded favorable results. Further study is needed to clarify the effect of concurrent chemoradiotherapy.

\section{REFERENCES}

[1] D. G. Pfister, S. A. Laurie, G. S. Weinstein, W. M. Mendenhall, D. J. Adelstein, K. K. Ang, et al., “American Society of Clinical Oncology Clinical Practice Guideline for the Use of Larynx-Preservation Strategies in the Treatment of Laryngeal Cancer," Journal of Clinical Oncology, Vol. 24, No. 22, 2006, pp. 3693-3704. doi:10.1200/JCO.2006.07.4559

[2] E. Cellai, P. Frata, S. M. Magrini, F. Paiar, R. Barca, S. Fondelli, et al., "Radical Radiotherapy for Early Glottic Cancer: Results in a Series of 1087 Patients from Two Italian Radiation Oncology Centers. I. The Case of T1N0 Disease," International Journal of Radiation Oncology *Biology*Physics, Vol. 63, No. 5, 2005, pp. 1378-1386. doi:10.1016/j.ijrobp.2005.05.018

[3] P. Frata, E. Cellai, S. M. Magrini, B. Bonetti, E. Vitali, S. Tonoli, et al., "Radical Radiotherapy for Early Glottic Cancer: Results in a Series of 1087 Patients from Two Italian Radiation Oncology Centers. II. The Case of T2N0 Disease," International Journal of Radiation Oncology *Biology*Physics, Vol. 63, No. 5, 2005, pp. 1378-1394. doi:10.1016/j.ijrobp.2005.05.013

[4] L. H. Sobin and C. Wittekind, "International Union against Cancer (UICC) TNM Classification of Malignant Tumours," 6th Edition, Wiley, New York, 2002.

[5] Eastern Cooperative Oncology Group (ECOG) Performance Status.

[6] A. H. Calvert, D. R. Newell, L. A. Gumbrell, S. O’Reilly, M. Burnell, F. E. Boxall, et al., "Carboplatin Dosage: Prospective Evaluation of a Simple Formula Based on Renal Function,” Journal of Clinical Oncology, Vol. 7, No. 11, 1989, pp. 1748-1756.

[7] National Cancer Institute, “Common Terminology Crite- ria for Adverse Events v4.0,”

http://ctep.cancer.gov/protocolDevelopment/electronicapplications/ctc.htm

[8] R Foundation for Statistical Computing, "Free Software," http://www.r-project.org

[9] E. L. Kaplan and P. Meier, "Non-Parametric Estimation from Incomplete Observations," Journal of the American Statistical Association, Vol. 53, No. 282, 1958, pp. 457 481. doi:10.1080/01621459.1958.10501452

[10] T. Akimoto, T. Nonaka, Y. Kitamoto, H. Ishikawa, H. Ninomiya, K. Chikamatsu, et al., "Radiation Therapy for T2N0 Laryngeal Cancer: A Retrospective Analysis for the Impact of Concurrent Chemotherapy on Local Control,” International Journal of Radiation Oncology*Biology* Physics, Vol. 64, No. 4, 2006, pp. 995-1001. doi:10.1016/j.ijrobp.2005.10.003

[11] Y. Kumamoto, M. Masuda, Y. Kuratomi, S. Toh, A. Shinokuma, K. Chujo, et al., "FAR" Chemoradiotherapy Improves Laryngeal Preservation Rates in Patients with T2N0 Glottic Carcinoma,” Head \& Neck, Vol. 24, No. 7, 2002, pp. 637-642. doi:10.1002/hed.10114

[12] Y. Itoh and N. Fuwa, "Retrospective Analysis: Concurrent Chemoradiotherapy Using Protracted Continuous Infusion of Low-Dose Cisplatin and 5-Fluorouracil for T2N0 Glottic Cancer," Radiation Medicine, Vol. 24, No. 4, 2006, pp. 277-281. doi:10.1007/s11604-005-1517-1

[13] M. Nakayama, K. Hayakawa, M. Okamoto, Y. Niibe, H. Ishiyama and S. Kotani, "Phase I/II Trial of Concurrent Use of S-1 and Radiation Therapy for T2 Glottic Cancer," Japanese Journal of Clinical Oncology, Vol. 40, No. 10, 2010, pp. 921-926. doi:10.1093/jjco/hyq077

[14] A. B. Karim, J. H. Kralendonk, L. Y. Yap, K. H. Njo, A. H. Tierie, R. M. Tiwari, et al., "Heterogeneity of Stage II Glottic Carcinoma and Its Therapeutic Implications,” International Journal of Radiation Oncology*Biology*Physics, Vol. 13, No. 3, 1987, pp. 313-317. doi:10.1016/0360-3016(87)90004-6

[15] S. P. Reddy, R. L. Hong, S. Nagda and B. Emami, “Effect of Tumor Bulk on Local Control and Survival of Patients with T1 Glottic Cancer: A 30-Year Experience,” International Journal of Radiation Oncology*Biology*Physics, Vol. 69, No. 5, 2007, pp. 1389-1394. doi:10.1016/j.ijrobp.2007.05.077

[16] E. D. McCoul and G. Har-El, "Meta-Analysis of Impaired Vocal Cord Mobility as a Prognostic Factor in T2 Glottic Carcinoma,” JAMA Otolaryngology-Head \& Neck Surgery, Vol. 135, No. 5, 2009, pp. 479-486. doi:10.1001/archoto.2009.47

[17] H. Haugen, K. A. Johansson and C. Mercke, "Hyperfractionated-Accelerated or Conventionally Fractionated Radiotherapy for Early Glottic Cancer,” International Journal of Radiation Oncology*Biology*Physics, Vol. 52, No. 1, 2002, pp. 109-119. doi:10.1016/S0360-3016(01)01812-0

[18] H. Tsuji, T. Kiba, M. Nagata, T. Inoue, H. Yukawa, T. Yamashita, et al., "A Phase I Study of Concurrent Chemoradiotherapy with S-1 for T2N0 Glottic Carcinoma," Oncology, Vol. 71, No. 5-6, 2006, pp. 369-373. doi:10.1159/000108385 